\title{
Role of Transdermal Glyceryl Trinitrate Patch versus Oral Isoxsuprine as a Tocolytic Agent in Preterm Labor
}

\author{
${ }^{1}$ Sangita N Ajmani, ${ }^{2}$ Vinita Sarbhai, ${ }^{3}$ Vertika Kishore
}

\begin{abstract}
Introduction: In India, $10-15 \%$ of all babies are born prematurely, and it accounts for $70-80 \%$ of all perinatal deaths. Beta-agonist like Isoxsuprine is widely used to prevent preterm labor, but can sometimes have life-threatening side effects; hence alternative glyceryl trinitrate (GTN) patch is compared with Isoxsuprine for efficacy and safety.
\end{abstract}

Methods: Fifty women of gestation age 28-36 weeks, with two or more uterine contractions every 10 minutes for more than 1 hour but not in active labor with cervical dilations less than $2 \mathrm{~cm}$. were included in the study. Twenty-five women were administered tablet isoxsuprine (duvadilan) $10 \mathrm{mg}$ thrice daily and the rest 25 women received transdermal GTN patch releasing $10 \mathrm{mg}$ per 24 hours. If after 2 hours there was no effect, an additional patch of $10 \mathrm{mg}$ was applied. Time taken for the arrest of contractions, dose required for cessation of contractions, duration of delay in delivery, foetal outcome, APGAR score at birth, and birth weight were recorded for both the groups.

Results: Eighty-eight percent achieved successful tocolysis in GTN group as compared to $76 \%$ in Isoxsuprine group ( $p=0.467$ ), while the mean time taken to stop uterine contractions was less in GTN group (4.84 hours) as compared to Isoxsuprine group (7.8 hours) $(p=0.020)$. Mean gestational age at delivery was 34.97 weeks in the GTN group versus 33.24 weeks in isoxsuprine group ( $p=0.004)$. Fetal outcome and maternal adverse effects was more favorable in the GTN group as compared to Isoxsuprine.

Conclusion: Nitroglycerine is well tolerated, noninvasive and convenient therapy for tocolysis, requiring minimal monitoring of mother and fetus as compared to Isoxsuprine.

Keywords: Glyceryl trinitrate patch, Isoxsuprine, Preterm labor, Tocolysis.

How to cite this article: Ajmani SN, Sarbhai V, Kishore V. Role of Transdermal Glyceryl Trinitrate Patch versus Oral Isoxsuprine as a Tocolytic Agent in Preterm Labor. J South Asian Feder Obst Gynae 2018;10(Suppl 2):405-408.

Source of support: Nil

Conflict of interest: None

Date of received: 15 January 2017

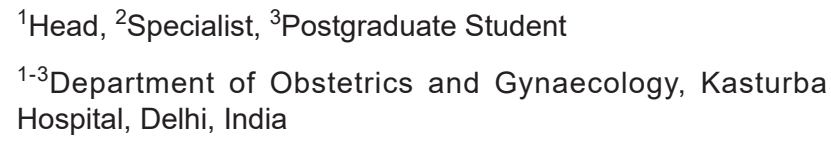

Corresponding Author: Vinita Sarbhai, Specialist, Department of Obstetrics and Gynaecology, Kasturba Hospital, Delhi, India, e-mail: vinitasarbhai@gmail.com
Date of acceptance: 8 July 2017

Date of publication: July 2019

\section{BACKGROUND}

Preterm labor results in an increase in the incidence of perinatal morbidity and mortality and remains a significant contentious issue in obstetrics. The incidence of preterm labor varies between $5 \%$ and $10 \%$. In India, $10-15 \%$ of all babies are born prematurely, and $70-80 \%$ of all perinatal deaths are due to prematurity. ${ }^{1,2}$

Preterm babies require special care and expensive equipment for their survival; therefore the mainstay in the management of preterm deliveries remains prolongation of gestation by various methods to stop preterm labor. Hence the focus of management is on tocolysis by drugs, and the additional aim is to buy time for the corticosteroids to cause an effective pulmonary maturation. The best tocolytic drug should have maximum efficacy with minimum immediate and delayed adverse effects. Isoxsuprine (duvadilan) and ritodrine are widely used but, being beta-agonists, they have a high frequency of undesirable and sometimes critical or potentially deleterious side effects for the antenatal women. In recent years there has been a lot of research focussed on finding a safer alternative.

Recently glyceryl trinitrate (GTN) in the form of transdermal patch has been tried as a tocolytic agent. Its chemical name is 1, 2, 3-Propanetriol trinitrate. In the patch, GTN impregnated polymer is present and it releases $20-25$ micrograms $/ \mathrm{cm}^{2} /$ per hour of GTN. GTN is nitric oxide (NO) donor, which is an endogenous diatomic molecule and acts as a potent smooth muscle relaxant. In preterm labor, NO synthetase activity decreases resulting in decreased NO production causing smooth muscle to contract. NO-donors, like the GTN patch, provide NO from outside, leading to relaxation of uterine muscles.

The objective of the present study was to study the efficacy and safety of GTN patch and compare it with conventional oral Duvadilan therapy in preterm labor.

\section{METHODS}

A prospective study of 50 pregnant women, admitted with preterm labour was conducted in the Department of Obstetrics and Gynaecology, Kasturba Hospital, Delhi. 
Women of gestation period of 28-36 weeks with two or more uterine contractions every 10 minutes, lasting for more than 1 hour, but not in active labor with cervical dilations less than $2 \mathrm{~cm}$, were included in the study. Those with obstetrical complications like multiple gestations, PROM, APH, PIH, previous LSCS, IUGR, and other medical complications like heart diseases, diabetes mellitus, severe anemia, and renal diseases were excluded from the study.

A detailed history was taken from the patients. General physical examination, obstetric examination, routine investigations, and ultrasounds were performed. The women were randomly assigned into two groups.

- Group $1(n=25)$ consisted of 25 women in preterm labor who received transdermal patch GTN patch application. Patch releasing $10 \mathrm{mg}$ per 24 hours was applied over the abdominal skin, below the umbilicus and the rate of contractions was monitored. If there was no reduction in interval or duration of contractions after 2 hours, another GTN patch of $10 \mathrm{mg}$ was applied. Patches remain for 24 hours, after cessation of contractions, at the end of which they were removed. The patients were asked to rest in the left lateral position and instructed not to get up suddenly from the bed from a supine position.

- Group-2 $(n=25)$ consisted of 25 women who were administered tablet isoxsuprine (duvadilan) $10 \mathrm{mg}$ thrice daily until contractions stopped. The two groups were matched for age, parity and cervical findings. All patients were given two doses of inj. dexamethasone $12 \mathrm{mg}$ intramuscularly,12 hours apart, to enhance lung maturity. After baseline examination patients were monitored for vitals, fetal heart rate, and uterine contractions, initially every 15 minutes for 1 hour and then at an hourly interval for 24 hours.

\section{RESULTS}

Following parameters were recorded. Time is taken for the arrest of contractions, the dose required for cessation for contractions, time period of prolongation of gestation, fetal outcome, APGAR score at birth, and birth weight. Statistical analysis of the data obtained was done.

Prolongation of gestation by 48 hours was considered to be successful tocolysis, 22 patients (88\%) achieved successful tocolysis in the GTN group as compared to 19 patients (76\%) in isoxsuprine group. However, there was no statistically significant difference $(p=0.467)$ in both groups with regards to the prolongation of pregnancy. The mean time is taken to stop uterine contractions in the GTN group (4.84 hours) was significantly lesser as compared to 7.8 hours in Isoxsuprine group $(p=0.020)$.

The mean duration of prolongation in our study was 12 days in the GTN group and 7 days in isoxsuprine group ( $p$ value -0.06 ). Among the successful cases, 17 patients required $10 \mathrm{mg}$ patch and 5 patients required 2 patches of $10 \mathrm{mg}$ each. Mean dose required to stop contractions in isoxsuprine group was $22.40 \mathrm{mg}$; range being from 10-30 mg (Table 1).

The fetal outcome was more favorable in the GTN group as compared to Isoxsuprine group. APGAR score at 1 minute was higher in the GTN group than in Isoxsuprine group. Eighteen neonates in the GTN group were transferred with mother versus 7 neonates in Isoxsuprine group. Nursery stay was because of prematurity in the GTN group (4 babies), while the cause of nursery stay in Isoxsuprine group was prematurity and meconium stained liquor. One neonate $(4 \%)$ has physiological jaundice in the GTN group. A much higher percentage of metabolic complication were seen in isoxsuprine group 3 $(12 \%)$ babies had apnea of prematurity, $6(24 \%)$ neonates had sepsis due to low birth weight. 6 (24\%) neonates had icterus, due to both physiologic and pathological causes. Mean gestational age at delivery was 34.97 weeks in the GTN group versus 33.24 weeks in isoxsuprine group $(p=0.004)$ (statistically significant) (Table 2$)$.

\section{Maternal Adverse Effects of the Drugs}

Very few adverse effects were noted in group 1 (GTN). The most common adverse effect was a headache which bothered 7 patients (28\%). It was mild and relieved by hydration, rest, reassurance and simple analgesic like paracetamol. None of the patients discontinued the therapy because of headache. The cutaneous reaction in the form of erythematous rash with burning sensation was seen in $4(16 \%)$ of patients, which got relieved on its own and no medication was needed for the same. No other side effects like tachycardia, hypotension or dizziness were seen. In the isoxsuprine groups following

Table 1: Analysis of successful cases

\begin{tabular}{llllll}
\hline & \multicolumn{2}{c}{ GTN (Group 1) N=22 } & \multicolumn{2}{c}{ Isoxsuprine (Group 2) $N=19$} & $p$ value \\
\cline { 2 - 5 } Parameter & Mean+SD & Range & Mean+SD & Range \\
\hline Time taken to stop contractions (hours) & $4.84+2.29$ & $1.5-8.45$ & $7.80-2.18$ & $4-16.10$ & 0.020 \\
Duration: Prolongation of pregnancy (days) & $12.07+8.03$ & $2.0-70$ & $7.02-5.02$ & $2.2-49$ & 0.06 \\
Dose required & $10 \mathrm{mg}-17 \mathrm{pts}$ & & & \\
& $20 \mathrm{mg}-5 \mathrm{pts}$ & $10-20$ & $22.40+12.68$ & $10-30$ \\
\hline
\end{tabular}


Role of Transdermal Glyceryl Trinitrate Patch versus Oral Isoxsuprine as a Tocolytic Agent in Preterm Labor

\begin{tabular}{|c|c|c|c|c|c|}
\hline \multicolumn{2}{|c|}{ Successful cases } & $\begin{array}{l}28-30 \\
\text { weeks }\end{array}$ & $\begin{array}{l}\text { 30-32 } \\
\text { weeks }\end{array}$ & $\begin{array}{l}\text { 32-34 } \\
\text { weeks }\end{array}$ & $\begin{array}{l}>34 \\
\text { weeks }\end{array}$ \\
\hline \multicolumn{2}{|c|}{ GTN group } & 5 & 7 & 7 & 3 \\
\hline \multicolumn{2}{|c|}{ Isoxsuprine group } & 7 & 6 & 5 & 1 \\
\hline \multicolumn{6}{|c|}{ Table 4: Success rate in isoxusprine group in various studies } \\
\hline S. no. & \multicolumn{2}{|l|}{ Author } & Year & \multicolumn{2}{|c|}{ Success rate (\%) } \\
\hline 1. & \multicolumn{2}{|l|}{ Singh $^{9}$} & 1987 & \multicolumn{2}{|l|}{85} \\
\hline 2. & \multicolumn{2}{|c|}{ Sphillicci et al. ${ }^{10}$} & 2000 & \multicolumn{2}{|l|}{90.24} \\
\hline 3. & \multicolumn{2}{|c|}{ Present study } & 2015 & \multicolumn{2}{|l|}{76} \\
\hline
\end{tabular}

adverse effects were seen-dizziness $(8 \%)$, palpitation $(20 \%)$, tachycardia $(24 \%)$. No other serious side effects like hypotension or pulmonary edema were seen.

\section{DISCUSSION}

Many modalities of halting labor pains in preterm pains are available, but none of them have been clearly shown to be superior to others. The main concern in cases of preterm labor is the maturity of lung and associated respiratory distress. Thus the aim of tocolysis is to delay the delivery for 48 hours to complete the course of steroid injection for enhancing lung maturity and improving the neonatal outcome. Nitroglycerine, a nitric oxide donor, is associated with infrequent maternal and fetal adverse effects and hence may be an effective choice as a tocolytic agent as compared to existing other agents like $\beta 2$ agonists, injection $\mathrm{MgSO}_{4}$, etc. ${ }^{11}$

The success rate of GTN patch was compared to other studies (Table 3). Rowlands et al. ${ }^{5}$ reported a success rate of $100 \%$ as GTN patch was applied till the uterine contraction stopped and continued till term.

Lees et al. reported a success rate of $92.3 \%$ and concluded that GTN patches are a very effective method for cessation of uterine contractions in preterm labor as it is safe, well-tolerated, and noninvasive method. ${ }^{3}$

Rowlands concluded that glyceryl trinitrate provides an effective and safe method of tocolysis. This is well suited for long-term use with success rate as $90 \%$ with average prolongation of pregnancy by 46.2 days. ${ }^{4}$

The success rate of Isoxsuprine group was compared to that of other studies in Table 4. It was found higher in other studies because they have used parenteral route initially followed by oral while we have used the only oral route.

Çalışkan et al. compared GTN patch and $\mathrm{MgSO} 4$ intramuscularly in 41 patients and found that uterine contraction cessation time was lower in women given GTN patch $(3.66 \pm 1.28$ hours $)$ as compared to $6.83 \pm 3.47$ hours for $\mathrm{MgSO} 4$ group. They observed that the GTN group had significantly lower maternal side effects than the $\mathrm{MgSO} 4$ group, and concluded that GTN effectively delays labor with less maternal adverse effects. GTN patch was found

Table 3: Success rate with GTN patch in various studies

\begin{tabular}{llll}
\hline S. No. & Author & Year & Success rate (\%) \\
\hline 1. & Lees et. al. $^{3}$ & 1994 & 92.3 \\
2. & Rowland et. al. $^{4}$ & 1996 & 90 \\
3. & Krishna et. al. $^{5}$ & 1996 & 100 \\
4. & Smith et. al. $^{6}$ & 1999 & 64.7 \\
5. & Habib $^{7}$ & 2014 & 88 \\
6. & Nankali $^{8}$ & 2014 & 87.5 \\
7. & Present study & 2015 & 88 \\
\hline
\end{tabular}

to be an acceptable, and safer alternative to injection $\mathrm{MgSO}_{4}$ which requires more intense monitoring also. ${ }^{12}$

Conde-Agudelo et al., in their meta-analysis of transdermal nitroglycerin versus $\beta 2$-adrenergic-receptor agonist for preterm labor, included data from 9 trials with a total of 1024 women. Women receiving transdermal nitro-glycerine patch application had a statistically significant lower rate of preterm birth as compared with women receiving $\beta$ agonists. A significant increase in the onset of therapy to delivery interval (MD 1.4 days, $95 \%$ CI 0.7-2.0; I $2=49 \%$ ) and baby birthweight (MD $331 \mathrm{~g}$, $95 \%$ CI 67-595; I 2 = 53\%) was also shown. ${ }^{13}$

The postponement of labor progression for more than 48 hours was taken as the criterion of successful therapy in the women receiving GTN patch by Humma Habib. This could be achieved in $66(88 \%)$ out of 75 patients. They concluded it to be a safe, cost-effective and reliable method of tocolysis. ${ }^{7}$

The main side effect reported was a headache as also observed by Wani et al. in $25 \%$ and total incidence of side effects with NTG to be $30 \% .{ }^{14}$

However, Nankali et al. have reported no difference between placebo and GTN in terms of successful tocolysis, and the mean prolongation of the pregnancy. ${ }^{8}$

\section{CONCLUSION}

From this study, it can be concluded that glyceryl trinitrate transdermal patch as a tocolytic agent is superior to beta-agonist Isoxsuprine hydrochloride. Nitroglycerine is not only more effective in prolongation of pregnancy and improving fetal outcome in preterm labor, but also safer for mother and fetus as compared to isoxsuprine hydrochloride group. It is well tolerated, noninvasive and convenient therapy for administration requiring minimal monitoring of mother and fetus.

\section{REFERENCES}

1. Rush R, Keirse M, et al. Contribution of preterm delivery to perinatal mortality. BMJ, 1976;2(6042);965-968.

2. Das B. A clinical study of preterm labour. J Obstet Gynaecol India 1986;(34):300. 
3. Lees C, Campbell S, et al. Arrest of preterm labour and prolongation of gestation with glyceryl trinitrate, a nitric oxide donor. The Lancet. 1994;343(8909):1325-1326.

4. Rowlands S, Trudinger B, et al. Treatment of Preterm Cervical Dilatation with Glyceryl Trinitrate, a Nitric Oxide Donor. The Australian and New Zealand Journal of Obstetrics and Gynaecology 1996;36(4):377-381.

5. Krishna U. Nitroglycerine skin patch application for tocolysis in premature onset of labour. Obs and Gynae today 1996;305307.

6. Smith G, Walker M, et al. Randomized Double-Blind PlaceboControlled Trial of Transdermal N itroglycerin for Preterm Labor. Obstetrical \& Gynecological Survey 2007;62(6):358-360.

7. Habib H, Sheikh GM. Transdermal nitroglycerine as a tocolytic in preterm labour. International Journal of Scientific and Research Publications 2014;4(6):1-4.

8. Nankali A, Kord Jamshidi P, et al. The Effects of Glyceryl Trinitrate Patch on the Treatment of Preterm Labor: A Single-blind Randomized Clinical Trial. J Repro d Infertil 2014;15(2):71-77.
9. Singh V, Sharma M, et al. Isoxsuprine and Premature Labour. Journal of Obsterics and Gynaeocology of India 1987, August, 485-488

10. Sphillicci MDB et al. Revista Brasileria De Medicina, Jan/Feb 2000;57(1/2)

11. Smith G, Walker M, et al. Randomised, double-blind, placebo controlled pilot study assessing nitroglycerin as a tocolytic. BJOG: An International Journal of Obstetrics and Gynaecology 1999;106(7):736-739.

12. Caliskan S, Narin M, et al. Glyceryl trinitrate for the treatment of preterm labor. Journal of the Turkish German Gynecological Association 2015;16(3):174-178.

13. Conde-Agudelo A, Romero R, et al. Nifedipine in the management of preterm labor: a systematic review and metaanalysis. American Journal of Obstetrics and Gynecology. 2011;204(2):134.e1-134.e20.

14. Wani M, Barakzai N, et al. Glyceryl trinitrate vs. ritodrine for the treatment of preterm labor. International Journal of Gynecology \& Obstetrics 2003;85(2):165-167. 